\title{
Caracterización geotécnica del perfil de los suelos lateríticos, Moa, Cuba
}

\author{
Amalia Beatriz Riverón Saldívar y José Alejandro Carménate Fernández \\ Universidad de Moa Holguín, Cuba \\ amabea1969@gmail.com, jacarmenatef@gmail.com
}

\begin{abstract}
RESUMEN
En este trabajo se presentan los resultados del estudio de las propiedades físicas y geotécnicas del perfil vertical de los suelos laterítico. El estudio de las propiedades físco-mecanicas se realiza de acuerdo al sistema unificado de clasificación de los suelos. La composición mineralógica se determina por DRx y la química por ICP-AES. En el estudio se analizan las diferentes zonas del perfil. Entre las propiedades físicas analizadas se encuentran la granulometría, peso específico de las partículas sólidas, densidad seca y porosidad, índice de poros, humedad y grado de saturación plasticidad, ángulo de fricción interna y cohesión. La capa superior ferricreta se caracteriza por una granulometría areno gravosa, mientras que en las zonas limonítica, transcición y saprolítica predomina la fracción limo-arcillosa.
\end{abstract}

Palablas claves: lateritas, limonitas, propiedades geotecnicas.

\section{Geotechnical characterization of the lateritic soil profile. Moa, Cuba.}

\begin{abstract}
This paper presents the results of the study of the physical and geotechnical properties of the vertical profile of lateritic soils. The study analyses the different zones of the profile. The study of the physical-mechanical properties is carried out according to the unified soil classification system. The mineralogical composition is determined by DRx and the chemical composition by ICP-AES. Among the physical properties analysed are particle size distribution, specific weight of solid particles, dry density and porosity, pore index, moisture and degree of saturation, plasticity, angle of internal friction and cohesion. The upper ferricretic layer is characterized by a sandy-gravelly grain size, whilst in the limonitic, transition and saprolitic zones the silt-clay fraction predominates.
\end{abstract}

Keywords: laterites; limonites; geotechnical properties. 


\section{Introducción}

Los suelos lateriticos de Cuba han sido estudiados considerando dos objetivos fundamentales: uno económico para la extracción del níquel y el cobalto y otro desde el punto de vista de su caracterización físico-mecánica para la construcción de obras civiles, infraestructuras y como materiales de construcción. En este trabajo centraremos la atención en el segundo aspecto, la caracterización físico-mecánica del perfil de los suelos lateríticos.

La corteza laterítica sobre rocas ultrabásicas (ofiolitas) se desarrolla en la zona norte de Cuba. En la figura 1 se muestran las áreas más representativas donde se encuentran los suelos lateríticos, estos ocupan el $7 \%$ de la superficie de la Isla de Cuba (Reeves et al., 1999). De las zonas señaladas las de mayor potencia del perfil y que representan un interés económico concreto por los elevados contenidos de $\mathrm{Ni}$ y Co se pueden agrupar en cuatro regiones: Provincia de $\mathrm{Pi}$ nar del Rió (región de Cajalbana), Provincia de Camagüey (Meseta de San Felipe) y dos regiones en la provincia de Holguín (Municipio de Mayarí y Municipio de Moa) (Figura 1). Estos depósitos, de acuerdo a la clasificación mineralógica de Brand et al. (1998), son depósitos de óxidos. Según Gleeson et al. (2001), el ejemplo mejor conocido a nivel mundial de depósitos de lateritas de Ni tipo óxidos es el de Moa (Moa Bay en literatura anglosajona). Debido a la magnitud de sus reservas y extensión dan lugar a un world-class deposits. Las reservas de mineral con contenido de $\mathrm{Ni}$ de valor económico ( $>0,9 \%$ en peso) se estiman en el orden de los 3000 millones de toneladas de Ni (UNI, 1994). De las cuatro regiones las más importantes y donde se realiza la explotación de estos yacimientos son Moa y Punta Gorda.

Hay que señalar que a lo largo de las más de los 58 años de actividad minera en la región de Moa, la población ha crecido de 2000 habitantes en el 1962 a más de 70 mil en 2020. Este aspecto ha conllevado el desarrollo de toda una infraestructura de carreteras, vivienda, acueductos, puerto, aeropuerto, dos complejos metalúrgicos y un combinado mecánico, asío como un gran número de escuelas que prestan servicio a uno de los renglones económicos más importante del país y que constituye la razón de ser del municipio Moa.

\section{Características generales del área de estudio}

El municipio de Moa se encuentra en el extremo oriental de la isla de Cuba, a $200 \mathrm{Km}$ de la ciudad de Holguín y a unos $1000 \mathrm{Km}$ de Ciudad de la Habana, la capital de la isla de Cuba. Constituye el municipio minero más importante del país, con una capacidad productiva superior a las 50000 toneladas por año de Ni (UNI, 1994) y 30000 toneladas por año de cromo (Proenza, 1998). Posee dos plantas metalúrgicas en explotación y una en construcción, puerto, aeropuerto y una población de 75000 habitantes. Toda la infraestructura de la industria minero-metalúrgica se localiza en la zona costera como se puede apreciar en la figura 3.

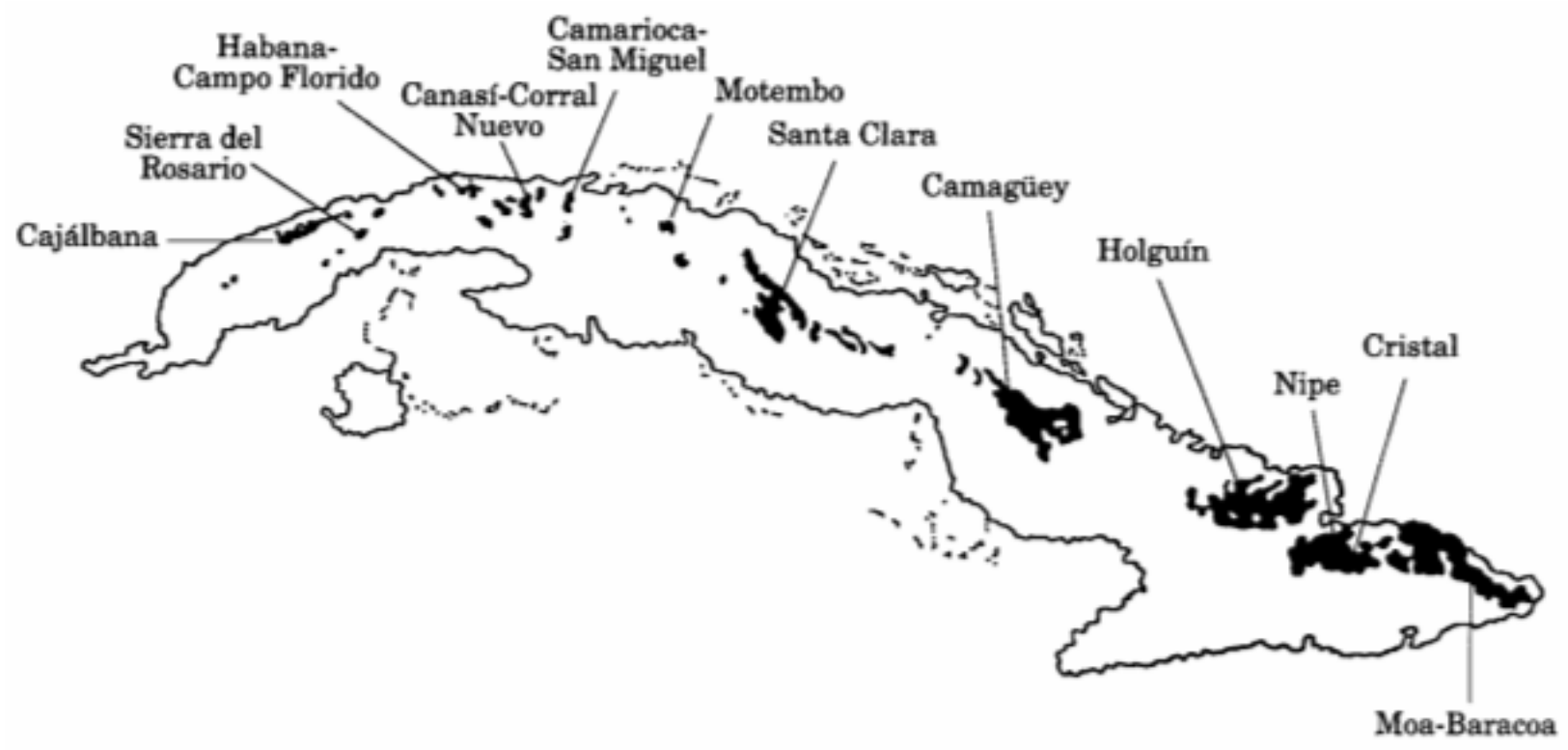

Figura 1. En color negro las zonas de distribución de las rocas ultramáficas y las principales áreas de afloramiento de los suelos lateríticos desarrollados sobre esta (Modificado de Reeves et al., 1999).

Figure 1. In black, the distribution zones of the ultramafic rocks and the main outcrop areas of the lateritic soils developed over it (Modified from Reeves et al., 1999). 


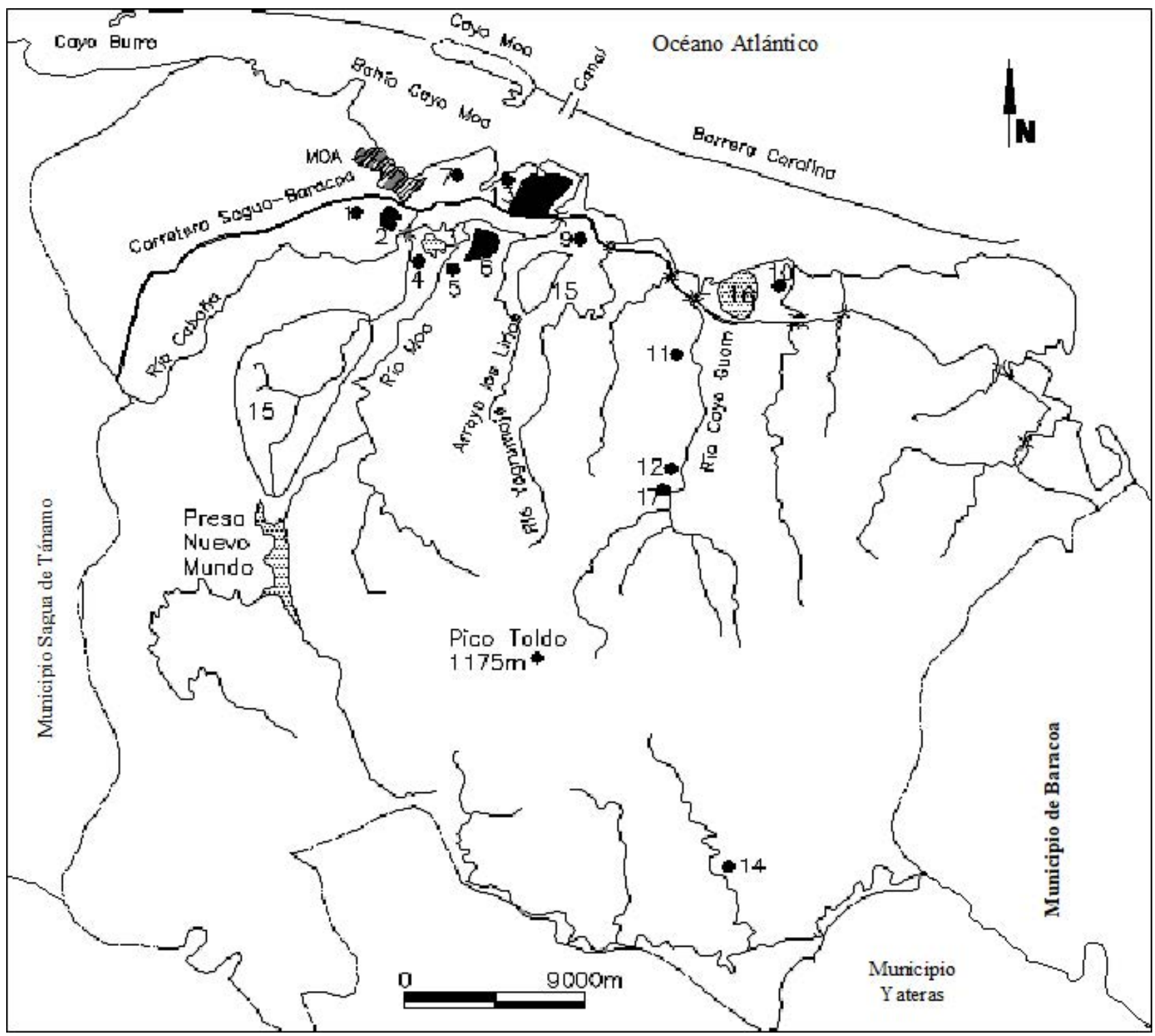

Figura 2. Principales instalaciones que conforman la infraestructura del distrito minero de Moa: 1) combinado mecánico, 2) presa de residuos inactiva, 3) embalse de agua, 4) planta metalúrgica de lixiviación ácida (SAL), 5) planta de tratamiento de agua, 6) presa de residuos en explotación planta metalúrgica $S A L, 7)$ laguna de oxidación, 8) presa de residuos en explotación planta $A C L, 9)$ planta metalúrgica de lixiviación con carbonato amoniacal $(A C L), 10)$ planta metalúrgica en construcción, 11) planta de beneficio del cromo, 13) puerto, 14) mina de cromo subterránea Mercedita en explotación, 15) minas de Ni y Co a cielo abierto, 16) presa de residuos en construcción, 12 y 17) mina de cromo (inactiva).

Figure 2. Main facilities that make up the infrastructure of the Moa mining district: 1) mechanical factory, 2) inactive tailings dam, 3) water reservoir, 4) acid leaching metallurgical plant $(S A L)$, 5) water treatment plant, 6) tailings dam in operation metallurgical SAL plant, 7) oxidation pond, 8) tailings dam in operation ACL plant, 9) ammonia carbonate leaching (ACL) metallurgical plant, 10) metallurgical plant under construction, 11) chrome beneficiation plant, 13) port, 14) Mercedita underground chrome mine, 15) open pit Ni and Co mines, 16) tailings dam under construction, 12 and 17) chrome mine (inactive).

\section{Geología}

El macizo ofiolítico Mayarí-Baracoa está compuesto por rocas ultrabásicas y básicas datadas como Jurásico-Cretácico inferior. Estas rocas ocupan más del $60 \%$ de la superficie del área de estudio, y tienen un espesor de hasta $1.000 \mathrm{~m}$. Los tipos litológicos son mayoritariamente harzburgitas (más de $70 \%$ ) y en menor medida dunitas. Todas ellas presentan un grado variable de serpentinización y en las zonas de cizallas y fracturas, puede llegar a ser mayor al $95 \%$ de la roca (típicas serpentinitas). Además, de estos tipos litológicos, se han descrito "peridotitas impregnadas" (con clinopiroxeno y plagioclasa), sills y diques de gabros, así como cuerpos de cromititas podiformes (Proenza 1999; Gleeson et al., (2003). 
Las harzburgitas, con texturas porfiroclásticas, se componen de olivino (70-90\%), ortopiroxenos (8-20\%), cromitas accesorias (1-2\%) y clinopiroxenos (hasta un $1 \%$ modal). Las dunitas presentan olivino (96-98\% modal), cromita accesoria (hasta un $3 \%$ ), y ortopiroxenos $(<2 \%)$. Todos estos minerales primarios están transformados a minerales secundarios (serpentina, clinocloro, magnetita, calcita, dolomita) en muy alta proporción. En menor cantidad ( $<1 \%$ modal), y asociados a los procesos de alteración, se pueden reconocer diferentes sulfuros de $\mathrm{Fe}-\mathrm{Ni}-\mathrm{Cu}$ (e.j: pirrotina, pentlandita, heazlewoodita, millerita, calcosina) y aleaciones de Fe-Ni (principalmente awaruíta). Sobre estas rocas ultramáficas serpentinizadas se desarrollan extensas cortezas lateríticas de $\mathrm{Fe}-\mathrm{Ni}$ Co (que albergan unas de las mayores reservas mundiales de $\mathrm{Ni} \mathrm{y} \mathrm{Co)} \mathrm{(Gleeson} \mathrm{et} \mathrm{al.,} 2003$ Lewis et al., 2006).

\section{Materiales y métodos}

Para la realización del trabajo se determinan las propiedades físico-mecánicas en un sondeo del corte laterítico de $14 \mathrm{~m}$. Este perfil incluye todas las zonas del perfil de suelo hasta la roca madre. El muestreo se realizó a intervalos de un metro. Las muestras fueron tomadas con un tomamuestras y almacenadas $y$ trasladadas al laboratorio hasta la realización de los análisis.

La separación de las muestras en las diferentes fracciones granulométricas se realizó por triplicado en cada una de las zonas del perfil del suelo laterítico. Tanto para los ensayos granulométricos como para la determinación de las otras propiedades físicas se utilizó el procedimiento establecido por la norma ASTM.

Para el ensayo granulométrico se utilizó la fracción menor de $10 \mathrm{~mm}$ Los intervalos granulométricos utilizados para el estudio son: $<0,032 \mathrm{~mm} ; 0,032-0,063 \mathrm{~mm}, 0,065-$ $0,125 \mathrm{~mm}, 0,125-0,250 \mathrm{~mm} ; 0,250-0,500 \mathrm{~mm} ; 0,5-1,0 \mathrm{~mm}$; $1,0-2,0 \mathrm{~mm} ;>2,0 \mathrm{~mm}$. Estos se obtuvieron por tamizado en húmedo. El material resultante de cada intervalo se dejó secar y luego se tomó una muestra para determinar su composición química siguiendo el procedimiento que se describe a continuación.

Mineralogía: la composición mineralógica por difracción de Rx. Ver detalles de la metodología en Rodríguez (2002).

Composición química de la fase sólida: para determinar la composición se tomaron $0.2 \mathrm{~g}$ de sólido y se secó en estufa a 40 grados. En las muestras se analizaron los elementos ( $\mathrm{Fe}, \mathrm{Cr}, \mathrm{Ni}, \mathrm{Co})$, por AAS e ICPAES, previa digestión total de las muestras por ataques sucesivos de $\mathrm{HF}, \mathrm{HNO}_{3}$ y $\mathrm{HClO}_{4}$. Para verificar los resultados de la concentración de los metales en los análisis se utilizó el patrón internacional de serpentina del Service D'Analyse des Roches et des Mineraux du CNRS de Francia. Este patrón permite corregir las desviaciones de la concentración de los diferentes elementos analizados. Los análisis se realizaron en el servicio científico técnico de la Universidad de Girona y la Universidad de Barcelona.

\section{Resultados}

\section{Composición mineralógica}

En la tabla 3 se muestra la composición mineralógica promedio de cada una de las zonas del corte laterítico. Se aprecia un predominio de la goethita en la parte superior del corte y de la serpentinita en la parte baja del mismo. En la figura 5 se muestran los diagramas de DRX de diferentes muestras del corte (1: Zona limo-

\begin{tabular}{|c|c|c|c|c|c|}
\hline Minerales & Formula Química & $\begin{array}{l}\text { Zona lim } \\
\text { Ferricreta } \\
\text { Limonita }\end{array}$ & onítica & $\begin{array}{l}\text { Zona de } \\
\text { transición }\end{array}$ & $\begin{array}{l}\text { Zona } \\
\text { saprolítica }\end{array}$ \\
\hline Óxidos e hidróxidos de hierro & Goethita $\mathrm{FeOOH}$, & $60-65$ & $60-70$ & $60-85$ & $15-35$ \\
\hline & Hematita $\left(\mathrm{Fe}^{2+} \mathrm{O}_{3}\right)$ & $2-5$ & $3-5$ & $2-4$ & \\
\hline $\begin{array}{l}\text { Minerales del grupo de la } \\
\text { serpentina }\end{array}$ & $\begin{array}{l}\text { Forsterita }\left(\mathrm{Mg}_{2} \mathrm{SiO}_{4}\right) \\
\text { Clorita }\left(\mathrm{H}_{4} \mathrm{Mg}_{3} \mathrm{Si}_{2} \mathrm{O}_{9}\right) \\
\text { Lizardita } \\
\mathrm{Mg}_{3}(\mathrm{SiAl})_{2} \mathrm{O}_{5} \mathrm{OH}_{4} \\
\text { Antigorita } \\
\left(\mathrm{Mg}_{6}\left(\mathrm{Si}_{4} \mathrm{O}_{10}\right)(\mathrm{OH})\right)\end{array}$ & & & $4-6$ & $25-30$ \\
\hline Espinelas & $\begin{array}{l}\text { Magnetita }\left(\mathrm{Fe}^{2+} \mathrm{Fe}_{2}{ }^{3+} \mathrm{O}_{4}\right) \\
\text { Maghemita }\left(\mathrm{Fe}^{3+} \mathrm{O}_{3}\right)\end{array}$ & $1-3$ & $3-4$ & $1-3$ & $1-2$ \\
\hline Gibbsita & $\mathrm{Al}(\mathrm{OH})_{3}$ & $12-20$ & $8-10$ & $3-5$ & $1-2$ \\
\hline Cromitas & & $2-3$ & $1-3$ & $1-2$ & 0.1 \\
\hline Minerales de manganeso & & $0.4-1.2$ & $0.3-1$ & $0.1-0.4$ & $0-0.2$ \\
\hline
\end{tabular}

Tabla 1. Composición mineralógica predominante por zona del corte laterítico (en \% en peso semicuantitativo). Table 1. Predominant mineralogical composition by zone of the lateritic zone (in semi-quantitative weight \%). 
nitita, 2: zona de transición y 3: zona saprolítica) considerando dos fracciones granulométricas. Se indican las fases minerales identificadas. Están presentes en el parte superior del corte para la fracción entre 2-80 micras la goethita, guibsita, magemita, mientras que para la fracción menor de 2 micras no se observan las dos últimas. En la zona de transición están presentes en la fracción entre 2-80 micras la goethita, serpentinita y guibsita, sin embargo, en la fracción menor de $2 \mathrm{mi}-$ cras no se observa la guibsita, lo que quiere decir que su concentración es menor del $1 \%$ en peso. En la zona saprolítica para la fracción entre 2-8 micras se identifican la serpentinita, goethita y magemita, mientras que en la fracción menor de 2 micras solo están la primera.

La descripción de los depósitos laterítico que se expone a continuación es el que se ha desarrollado sobre las rocas ultramáficas (peridotitas y harzburguitas serpentinizadas) en la región de estudio. El perfil puede variar sus características en función de la composición petrológica del área del macizo ofiolítico en que se encuentre y del grado de desarrollo del perfil laterítico, así como de los procesos geológicos que hayan tenido lugar en el área, tales como erosión y redeposición de las cortezas lateríticas. Considerando estos aspectos se pueden reconocer en Cuba tres tipos de perfiles del suelo laterítico: In situ, redepositado, y sedimentario (continental y marino) (Formell and Buguelskiy, 1974; Formell, 1979; Formell y Oro, 1980; Rodríguez, 2002). En la figura 4, se puede observar una representación de cada uno de estos.

Como hemos señalado en los objetivos de este trabajo solamente se analizará el perfil de suelo "in situ". Sobre la zonación del corte laterítico se han escrito diferentes clasificaciones entre las que se encuentran: I) Linchenat and Shirokova, 1964, II) Lavaut 1998, III) Rodríguez, 2002). En nuestro trabajo nos basaremos en la última clasificación. De acuerdo a esta en el corte se distinguen 4 zonas que caracterizaremos a continuación.

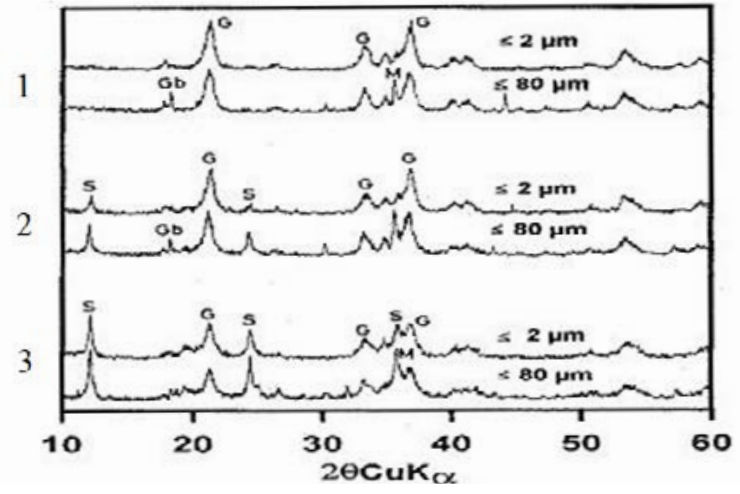

Figura 3. Diagramas de DRX de las muestras 1: Zona limonita, 2: zona de transición y 3: zona saprolítica para dos fracciones granulométricas. Se indican las fases identificadas: G: goethita, S: serpentinita, Gb:= guibsita, M: magemita.

Figure 3. XRD diagrams of samples 1: limonite zone, 2: transition zone and 3: saprolitic zone for two grain size fractions. The identified phases are indicated: G: goethite, S: serpentinite, Gb:= guibsite, M: magemite, S: serpentinite.

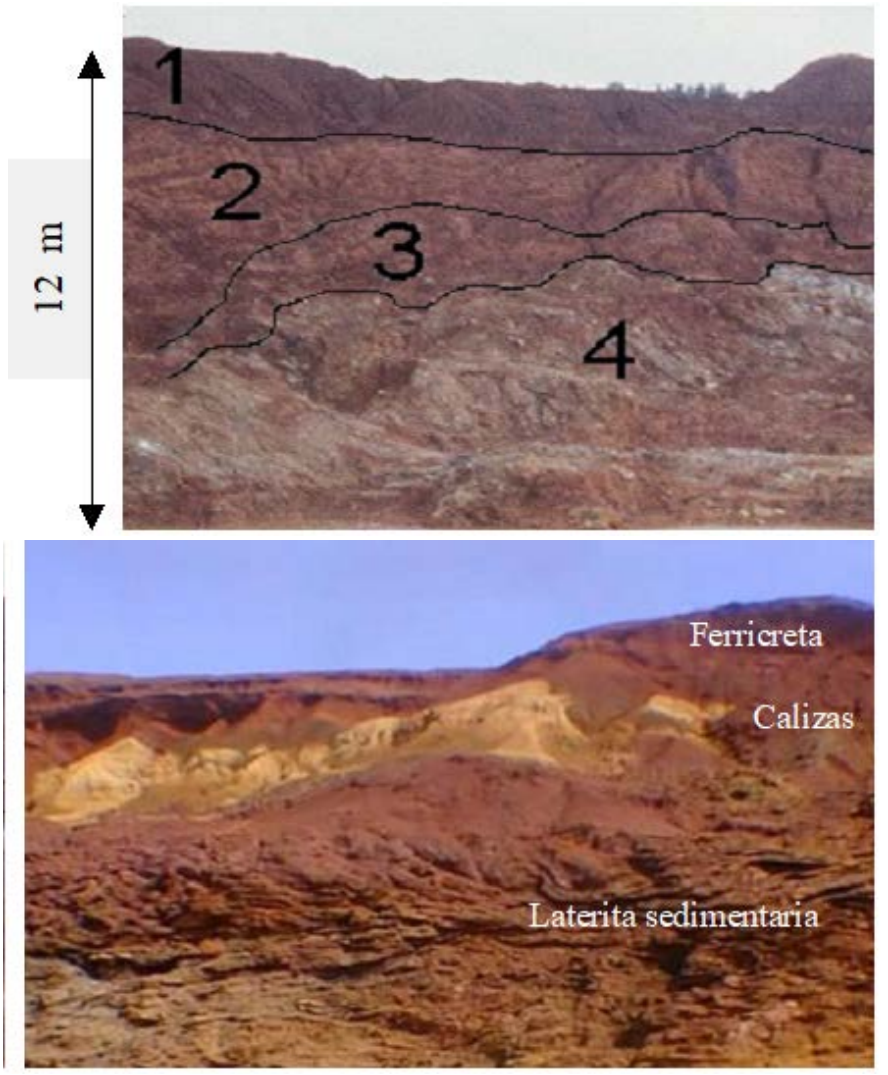

Figura 4. A la derecha corte laterítico in situ: 1) zona limonitita superior con ferricreta, 2) zona limonitita, 3) zona de transición, a) zona saproplítica y a la izquierda un corte de laterítica sedimentaria, donde se puede observar una intercalación de roca carbonatada de color más claro indicativa de una cuenca sedimentaría.

Figure 4. On the right, in situ laterite section: 1) upper limonite zone with ferricrete, 2) limonite zone, 3) transition zone, a) saproplite zone and on the left a sedimentary laterite section, where a lighter coloured carbonate rock intercalation can be observed, indicative of a sedimentary basin.

1- Zona limonítica superior: presenta un color marrón oscuro, con tonalidades negras. En la parte superficial se observan partículas de forma esférica de hidróxidos de $\mathrm{Fe}$, frecuentemente cementadas entre sí por material ferruginoso, de composición similar al que forman los propios hidróxidos, estos procesos de cementación dan lugar al crecimiento de capas de hidróxidos de hierro de variadas dimensiones, que pueden tener varias toneladas de peso. Estas capas son las que se conocen en la literatura como "ferralitas o ferricretas". El proceso de cementación de los hidróxidos de hierro es el resultado de los procesos a los que está expuesto el corte laterítico en condiciones naturales, debido a las variaciones climáticas anuales. La potencia es variable entre 0.2-15 m. La fracción arena puede llegar al $5 \%$. El pH del agua de poros es ligeramente ácido (5.1-6.5) y el agua es bicarbonatada-magnésica. La mineralogía está compuesta por óxidos e hidróxidos de hierro (Tabla 1). Su contenido medio elemental es: $\mathrm{Ni}$ inferior al $0.9 \%$, Fe entre $35 \mathrm{y}$ $50 \%, \mathrm{Mg}$ del 1 al $5 \%$, Cr entre 1 y $3 \%$, Al entre 5 y $10 \%$, 
Mn inferior al 1\%, Co entre el 0.01 y $0.07 \%$. Se pueden encontrar además pequeñas concentraciones de $\mathrm{Cu}$, $\mathrm{Zn}, \mathrm{V}$ yTi.

2- Zona limonítica inferior: se caracteriza por un color ocre o marrón oscuro. Su potencia es variable 2-6 $\mathrm{m}$. Presenta una humedad mayor que la zona superior. La granulometría es limo-arcillosa, predominando la fracción limo. El pH del agua de poros en esta capa es ligeramente ácido entre 6.2-6.5 y el agua es bicarbonatada-magnesica. Presenta una composición mineralógica y química similar a la superior. En esta zona se aprecia una disminución del contenido de Al y Fe y un incremento de Si, Mg y Ni (Figura 2). La principal diferencia respecto a la zona anterior es su contenido de níquel y la granulometría del material. El contenido de $\mathrm{Ni}$ es de 0.8-1.5\%, Fe 35-45\%, Mg 0.1$5 \%$, Si de $2-5 \%$.

3- Zona de transición: constituye la zona de transición entre la zona limonítica y la saprolítica. La coloración del corte es pardo-amarilla. Su granulome- tría es limo-arcillosa, con predominio de la fracción limo. La potencia media de esta zona es variable entre 5-10 m. En su interior se pueden encontrar bloques de la zona saprolítica. Esta zona se corresponde con la zona de variación del nivel freático del agua durante las diferentes estaciones del año (ciclos de secado y humedecimiento), aspecto que favorece la hidratación, disolución, transporte y precipitación de los diferentes elementos o compuestos químicos, así como el desarrollo de los procesos de oxidación de los minerales por la entrada de los diferentes gases atmosféricos (principalmente oxígeno) al bajar el nivel freático. El pH de esta capa es prácticamente neutro (6.8-7.1) y el agua es bicarbonatada-magnésica. La mineralogía es de óxidos e hidróxidos de $\mathrm{Fe}, \mathrm{Mg}, \mathrm{Al}$, Mn y minerales del grupo de serpentinas (Tabla 1). La concentración de níquel en esta zona es generalmente superior a $1.2 \%$, con rangos entre 1.2 y $3.2 \%$. El contenido de $\mathrm{Mg}$ es variable entre un 3-10\%, Fe entre un $25-35 \%$.

\begin{tabular}{llll}
\hline Partículas & Tamaño & Partículas & Tamaño \\
\hline Bloques & $>30 \mathrm{~cm}$ & Arena & $0.06 \mathrm{a} 2 \mathrm{~mm}$ \\
Bolos & $15 \mathrm{a} 30 \mathrm{~cm}$ & Limo & $0.002 \mathrm{a} 0.06 \mathrm{~mm}$ \\
Grava & $2 \mathrm{a} 15 \mathrm{~cm}$ & Fracción arcillosa & $<0.002 \mathrm{~mm}$ \\
& & $*$ Coloides & $<0.00045 \mathrm{~mm}$ \\
\hline
\end{tabular}

Tabla 2. Dimensiones de las partículas según su rango de tamaño de acuerdo con el sistema unificado de clasificación de los suelos (Lambe and Whitman, 1990). El * indica que no está en la bibliografía original.

Table 2: Particle dimensions by size range according to the unified soil classification system (Lambe and Whitman, 1990). The ${ }^{*}$ indicates that it is not in the original bibliography.

\section{$\rightarrow$ Limonita superior $\rightarrow$-Limonita inferior $\rightarrow$-Ferricreta $\rightarrow$ Saprolita}

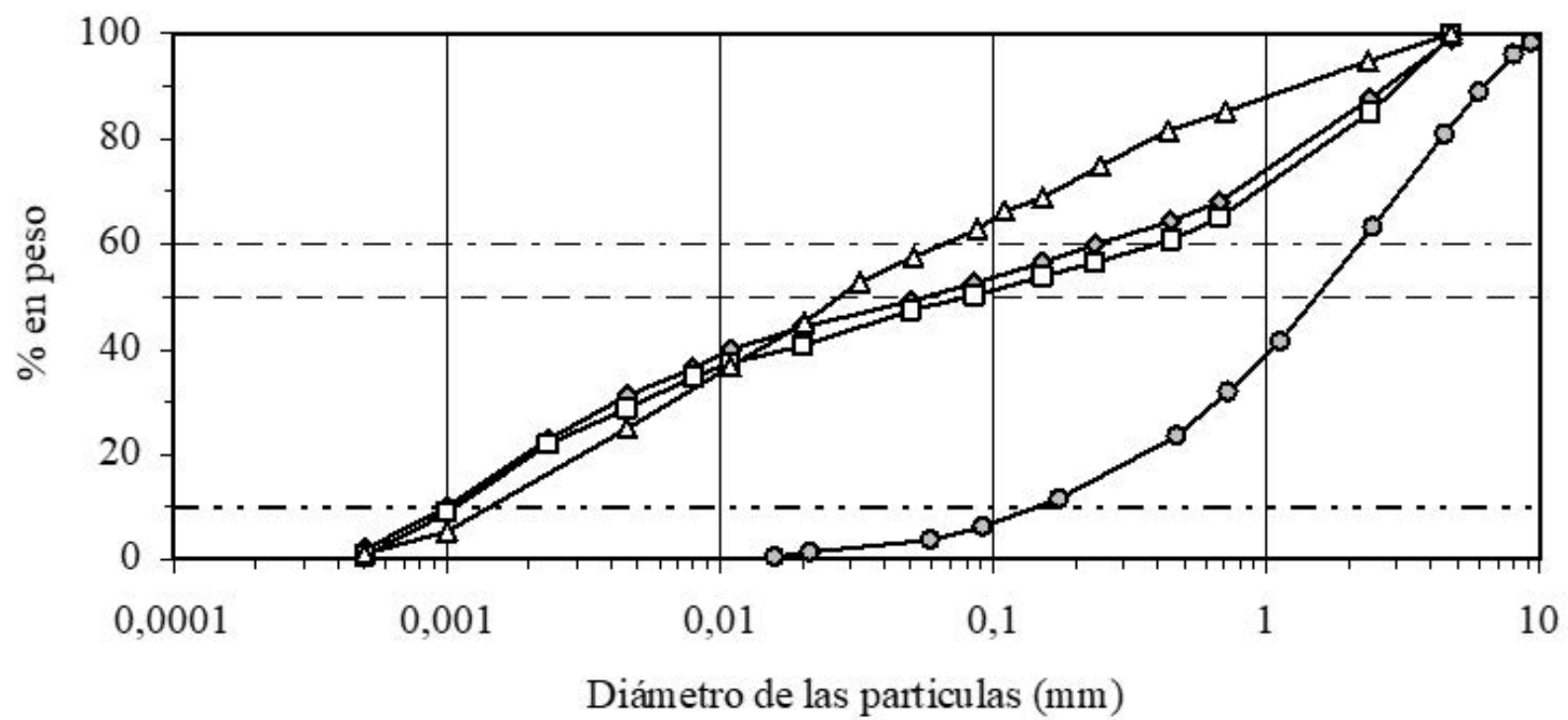

Figura 5. Curva granulométrica de las diferentes zonas del corte laterítico realizada con la distribución promedio de cuatro muestras. Figure 5. Granulometric curve of the different zones of the lateritic soil profile made with the average distribution of the four samples. 
4- Zona saprolítica: la coloración verde-amarilla varía en relación con su grado de alteración. Esta zona presenta mayor irregularidad en cuanto a su extensión y potencia, con un valor medio entre los 4-10 $\mathrm{m}$. Normalmente el material se encuentra en estado saturado. La granulometría es de tipo limo-arcilloso, predomina la fracción limo en más del $50 \%$ de su peso. Está compuesta por peridotitas y harzburguitas serpentinizadas muy meteorizadas. El agua es bicarbonatada-magnésica con valor de $\mathrm{pH}$ neutro a ligeramente básico entre 7.0-8.2. Mineralógicamente predominan los minerales del grupo de la serpentina (Tabla 1). Esta zona va pasando de su forma meteorizada a las rocas ultramáficas agrietadas. El contenido de níquel en esta zona es del orden de $1.5-3 \%$, el Fe entre el $10-25 \%$, el $\mathrm{Mg} 10-20 \%$, Si entre 20 y $30 \%$ (Figura 2 ).

De las capas del corte laterítico se utilizan industrialmente las zonas limonítica inferior, la de transición y la saprolítica. Las dos primeras zonas se emplean en el proceso metalúrgico de lixiviación con ácido sulfúrico (SAL) y las tres zonas en el proceso de lixiviación con carbonato amoniacal (ACL). La zona limonítica superior es el material que en el proceso de explotación de los yacimientos lateríticos forma los escombros o estériles.

\section{Características físico-mecánicas de los suelos laterí- ticos}

Granulometría: de acuerdo a los resultados de los ensayos de granulometría se puede apreciar que en el corte laterítico están presente diferentes clases granulométricas de acuerdo con el sistema internacional de clasificación de los suelos (Tabla 2, Fig. 5). En la parte superior del corte predomina la fracción arenosa y en menor medida las gravas (Figura 3). En las otras zonas del corte en profundidad predomina la fracción limo en más de un $40 \%$ y en menor medida se aprecia una proporción de arcillas que puede llegar hasta el $26 \%$. Estos resultados son coherentes con Almaguer (1995) y Almaguer y Zamarzry (1993).

Peso específico de las partículas sólidas: se puede apreciar como este decrece con la profundidad. EI mismo presenta un amplio rango de variación con valores entre $4,11-2,16 \mathrm{~g} / \mathrm{cm}^{3}$, este valor es muy superior al de los suelos naturales desarrollados sobre otro tipo de rocas. Su alto valor se debe a la presencia e altos contenidos de hierro y en menor medida cromo y otros metales.

Densidad natural: la densidad natural al igual que la seca decrece con la profundidad (Figura 4c). Esta propiedad se caracteriza por ser variable en el tiempo pues depende del contenido de humedad del suelo.

Densidad seca: es un parámetro que presenta un rango de variación mucho menor que el de las partículas sólidas. Se puede observar una disminución de esta con la profundidad (Figura $6 \mathrm{~b}$ ). Su valor al igual que el peso específico de las partículas sólidas no varía fácilmente en el tiempo.
Porosidad: aunque se incrementa relativamente con la profundidad en la zona correspondiente a la limonita y zona de transición, se puede observar que su variabilidad en profundidad no es muy grande. Los mayores valores están asociados a las zonas limonitita y de transición. Es un parámetro que, para un mismo perfil no varía rápidamente en el tiempo, aunque en el corte laterítico puede presentar una gran variación espacial debido a la heterogeneidad de este (Figura 46).

Índice de poros: es una propiedad directamente relacionada con la porosidad. Se aprecia un comportamiento irregular con un amplio rango de variación entre 1,03 y 2,4 . Los mayores valores se encuentran en la zona limonitita y los menores en la zona saprolítica y la parte superficial la ferricreta.

Humedad: es un parámetro que es variable en el tiempo. De acuerdo con la figura 4 e se puede observar que se incrementa con la profundidad (Fig. 6e). Los mayores valores los encontramos asociado a la zona de transición y saprolítica, mientras que los menores a la parte superior del corte, pues esta zona es la que está expuesta a los procesos de secado debido a la evaporación y el viento.

Grado de saturación: incrementa su valor con la profundidad (Figura 6f). En la parte baja del corte el material está completamente saturado. Este es un parámetro variable en el tiempo al igual que la humedad y está afectado mayoritariamente por la variación en la recarga debido a la infiltración del agua de lluvia o el flujo regional.

En la tabla 3 se muestra un resumen de las principales propiedades mecánicas del perfil del suelo laterítico considerando las diferentes zonas de desarrollo de la corteza de meteorización.

Limite liquido: la capa ferricreta posee un bajo limite líquido lo que la clasifica como no plástica. La capa limonitica posee una plasticidad emdia del 40 lo que la califica como un limo. La saprolitica posee un límite liquido superior a 50 lo que la califica como un material limo-arcilloso (Tabla 3).

Limite plástico: con respecto a la plasticidad son materiales de plasticidad media a baja, siendo la capa más plástica la zona limonitica. Le sigue con muy poca diferencia la capa saprolitica y con mayor diferencia la capa farricreta (Tabla 3 ).

Índice de plasticidad: considerando el índice de plasticidad de cada una de las capas no se aprecia una diferencia significativa siendo al que mayor valor presenta la capa saprolite seguida de la saprolite y en tercer lugar la limonita.

Cohesión: la cohesión presenta valores muy bajos lo que es coherente con el tipo de material que presenta bajo nulo contenido de minerales arcillosos (Tabla 1 y Figura 3 ).

Ángulo de fricción interna: el ángulo de fricción interna es variable alcanzando valores de 33 grados en la frricreta y de 18 en la zona limonitica. La variabilidad del ángulo de fricción interna se debe a el grado de desarrollo de 
Densidad de las partículas $\left(\mathrm{g} / \mathrm{cm}^{3}\right)$

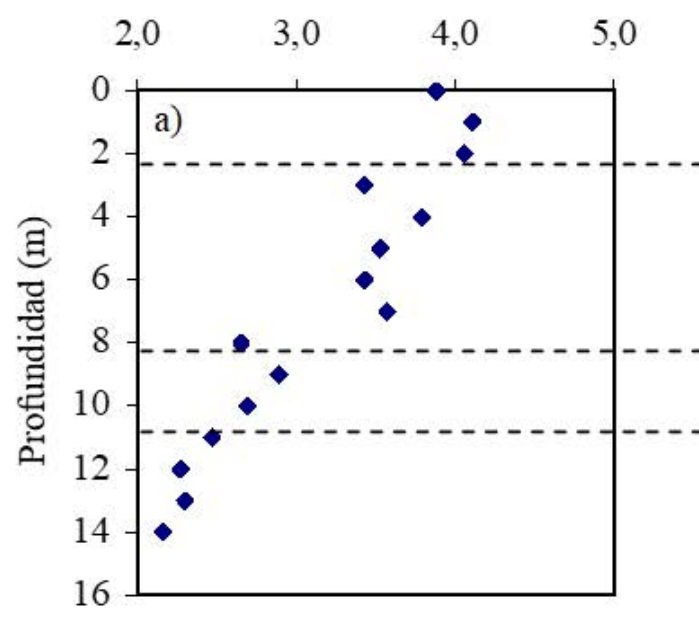

Densidad seca $\left(\mathrm{g} / \mathrm{cm}^{3}\right)$

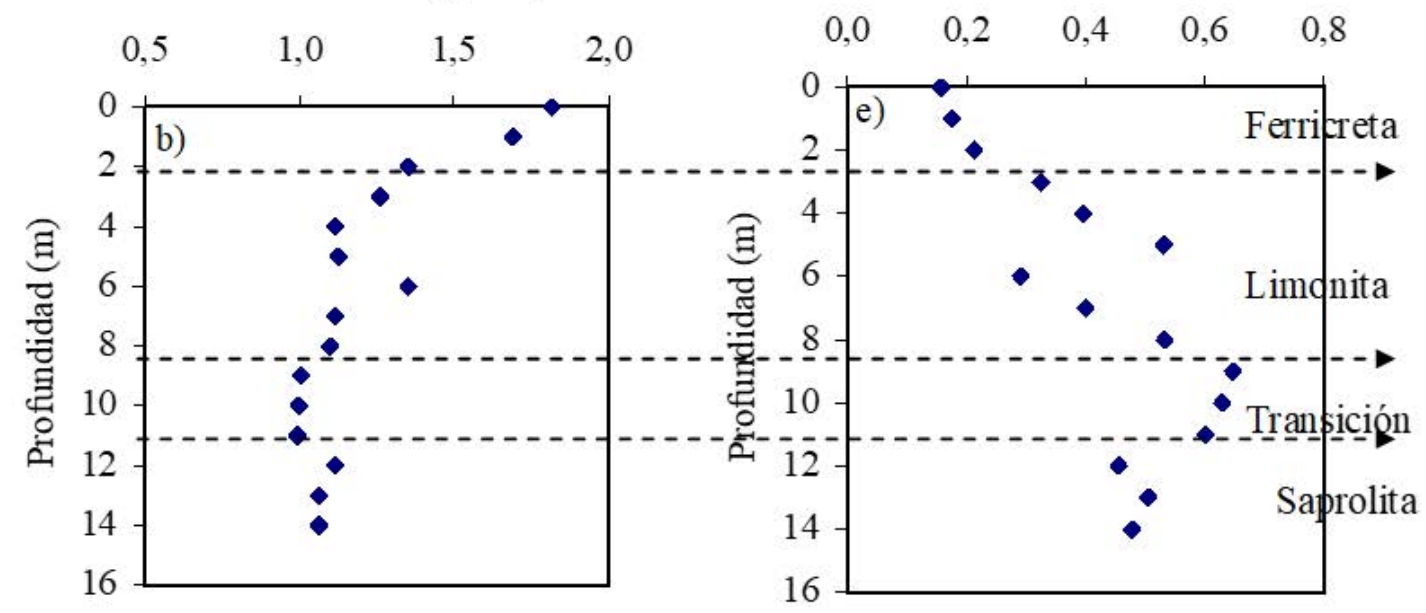

Densidad natural $\left(\mathrm{g} / \mathrm{cm}^{3}\right)$

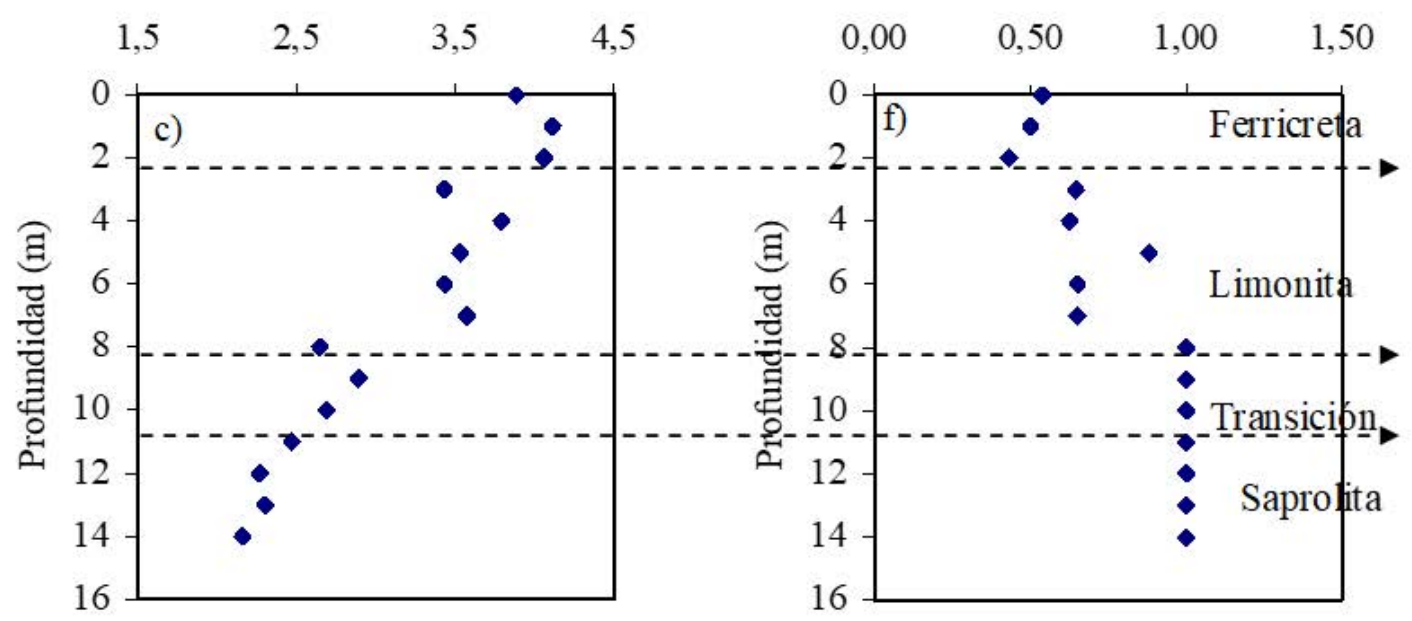

Figura 6. Propiedades físicas de los suelos lateríticos vs la profundidad del perfil.

Figure 6. Physical properties of lateritic soils profile vs. depth.

Porosidad

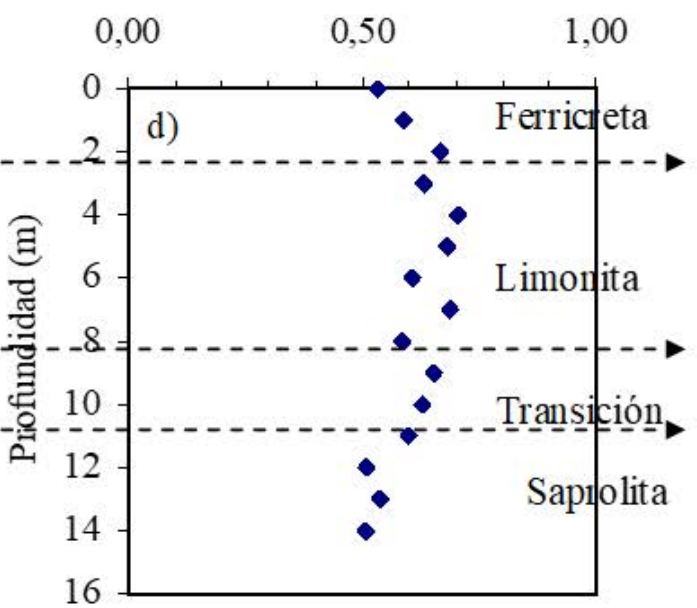

Humedad

Grado de saturación 


\begin{tabular}{|c|c|c|c|c|c|c|c|c|c|c|c|}
\hline & \multicolumn{11}{|c|}{ Parámetros } \\
\hline Ferricreta & LL & LP & IP & $\begin{array}{l}\mathrm{G}_{\mathrm{S}} \\
\left(\mathrm{g} / \mathrm{cm}^{3}\right)\end{array}$ & $\begin{array}{l}\mathrm{G}_{\mathrm{F}} \\
\left(\mathrm{g} / \mathrm{cm}^{3}\right)\end{array}$ & $\begin{array}{l}\mathrm{G}_{\mathrm{D}} \\
\left(\mathrm{g} / \mathrm{cm}^{3}\right)\end{array}$ & $\begin{array}{l}\mathrm{W}_{\mathrm{n}} \\
(\%)\end{array}$ & $\mathrm{e}$ & $\begin{array}{l}S \\
(\%)\end{array}$ & FI & $\begin{array}{l}\mathrm{C} \\
(\mathrm{kPa})\end{array}$ \\
\hline No. de muestras & 43 & 66 & 66 & 66 & 66 & 66 & 66 & 66 & & 37 & 60 \\
\hline Media & 4,7 & 46,6 & 31,6 & 15,4 & 3,7 & 2,2 & 1,8 & 26,8 & 1,1 & 27,1 & 28,6 \\
\hline Máxima & 13,0 & 78,0 & 53,0 & 43,0 & 4,0 & 3,3 & 2,4 & 83,3 & 3,4 & 35,1 & 39,0 \\
\hline Mínima & 0,3 & 30,0 & 21,0 & 7,0 & 3,2 & 1,6 & 0,8 & 12,9 & 0,5 & 15,0 & 28,0 \\
\hline Limonita & & & & & & & & & & & \\
\hline N. de muestras & 66 & 66 & 66 & 66 & 66 & 66 & 66 & 66 & 66 & 60 & 60 \\
\hline Media & 72,1 & 42,5 & 29,0 & 3,5 & 1,9 & 1,2 & 57,6 & 2,1 & 92,2 & 18,7 & 40,5 \\
\hline Máxima & 141,0 & 86,0 & 74,0 & 3,9 & 3,6 & 2,0 & 93,5 & 3,6 & 100,0 & 34,0 & 43,1 \\
\hline Mínima & 40,0 & 23,0 & 12,0 & 2,8 & 1,5 & 0,5 & 18,7 & 0,8 & 10,0 & 16,0 & 0,1 \\
\hline Saprolita & & & & & & & & & & & \\
\hline N. de muestras & 36 & 36 & 36 & 41 & 39 & 40 & 38 & 32 & 31 & 31 & 30 \\
\hline Media & 83,7 & 83,7 & 36,5 & 2,9 & 1,7 & 1,0 & 64,0 & 2,2 & 91,6 & 21,3 & 33,5 \\
\hline Máxima & 155,0 & 71,0 & 66,0 & 3,7 & 3,8 & 1,6 & 107,0 & 3,9 & 100,0 & 34,0 & 31,5 \\
\hline Mínima & 33,0 & 23,0 & 9,0 & 2,3 & 1,4 & 0,5 & 20,3 & 0,7 & 77,0 & 19,0 & 0,2 \\
\hline
\end{tabular}

Tabla 3. Propiedades físico-mecánicas de los suelos lateríticos y su rango de variación. LL: límite líquido, LP: límite plástico, IP: índice de plasticidad, $\mathrm{G}_{\mathrm{s}}$ : densidad de las partículas sólidas, $\mathrm{G}_{\mathrm{F}}$ : densidad natural, $\mathrm{G}_{\mathrm{D}}$ : densidad seca, $\mathrm{w}_{\mathrm{n}}$ : humedad natural, e: índice de poros, $\mathrm{Fl}$ : ángulo de fricción interna, C: cohesión.

Table 3. Physico-mechanical properties of lateritic soils and their range of variation. LL: liquid limit, LP: plastic limit, IP: plasticity index, $G_{S}$ : density of solid particles, $G_{F}$ : natural density, $G_{D}$ : dry density, $w_{n}$ : natural moisture, e: void ratio, Fl: angle of internal friction, $C$ : cohesion.

\section{Riesgo ambiental}

El principal riesgo ambiental que presentan los suelos lateríticos de la región de Moa es la erosión hídrica debido a la deforestación que desarrolla la minería a cielo abierto (Lavaut 1998, Rodríguez, 1991; Rodríguez, 2002). La fina granulometría y la baja cohesión de los materiales unido a su alta concentración de metales ha provocado la contaminación del río Moa y la Bahía. El aporte de sedimentos debido a la erosión hídrica es tan importante que ha sido necesario dragar el canal de acceso al puerto para poder facilitar el acceso de los barcos.

\section{Conclusiones}

Los ensayos de granulometría permiten diferenciar varias clases granulométricas. La zona superior ferricreta se caracteriza por una granulometría areno gravosa, mientras que en las zonas limonítica, transcición y saprolítica predomina la fracción limo-arcillosa. Las propiedades físicas muestran una gran variabilidad con la profundidad del perfil del suelo laterítico. En el caso de las propiedades mecánicas no se aprecia correlación significativa con ninguna de las propiedades físicas analizadas. Considerando estos resultados es posible señalar que hay que tener en cuenta el peso de las partículas sólidas y la granulometría del material a la hora de diferenciar las zonas del corte laterítico como elementos más significativos.

\section{Agradecimientos}

El presente trabajo se desarrolla dentro del proyecto “Mineralogía y geoquímica de $\mathrm{Ni}$, Co y elementos del grupo del platino (EGP) en lateritas niquelíferas: Referencia: CGL2006-07384/BET financiado por el Ministerio de Educación y Ciencia de España.

\section{Referencias}

Almaguer, A. (1995). Corteza de intemperismo. Algunas características de sus partículas finas. Minería y Geología. 12(1) ,9-19.

Almaguer, A. y Zamarzry (1993). Estudio de la distribución del hierro, níquel y cobalto en los tamaños de los granos que componen el perfil de las cortezas de intemperismo de las rocas ultramáficas hasta su desarrollo laterítico y su relación con la mineralogía. Minería y Geología. 2(2):17-23.

ASTM (Annual Book of ASTM Standards) (1993). Section 4. Construction. Volume 04.08. Soil and rock. Dimension stone; geosynthetics. $1296 \mathrm{p}$.

Formell , F. and Buguelskiy, Y.Y. (1974). Sobre la existencia de lateritas ferroniqueliferas redepositadas sobre calizas. Contribución a la geología de Cuba. Academia de Cencias, la Habana, 117-139.

Formell, F. (1979). Clasificación morfogenética de de las cortezas de interperismo sobre las rocas ultrabásicas de Cuba. Ciencia de laTierra y el Espacio, 1, 33-49. 
Formell, F. y Oro, J.R (1980). Sobre los procesos de redeposición del Yacimeinto Punta Gorda. Ciencia de laTierra y el Espacio, 2:53-66.

Gleeson S. A., Butt C. R. M., Elias M. (2003). A review. SEG Newsletter 54, 11-18.

Lambe, T.W. and Whitman, 1990. Mecánica de suelos. Editorial Limusa. México. $582 \mathrm{p}$.

Lavaut, W. (1998). Tendencias geológicas del intemperismo de las rocas ultramáficas en Cuba oriental. Minería y Geología, 15(1), 9-16.

Lewis J.F., Draper G., Proenza J.A., Espaillat, J. and Jiménez, J. (2006). Ophiolite-related ultramafic rocks (serpentinites) in the Caribbean Region: a review of their occurrence, composition, origin, emplacement and $\mathrm{Ni}$-laterite soil formation.

Linchenat, A. and Shirokova, I. (1964). Individual characteristics of the nickeliferous iron (laterite) deposits of the northeastern part of Cuba (Pinares de Mayari, Nicaro and Moa): Internat. Geol. Cong., 24 Montreal 1964. pt. 14, sec. 14, p. 172-187.

Proenza, J., Gervilla, F., Melgarejo. J. and Bodinier,
J.L. (1999). Al and Cr rich chromites from the Mayarí Baracoa ophiolitic belt (Eastern Cuba): consequence of interaction between volatile rich melts and peridotites in suprasubduction mantle, Economic Geology, 94, 547-566.

Rodríguez, A. (1991). Cambio de intervalo de perforación para la exploración detallada del yacimiento ferroniquelífero cobaltífero de Levisa. Minería y Geología. 8(1-3) 11-18.

Rodríguez, R. (2002). Estudio experimental de flujo y transporte de cromo, níquel y manganeso en residuos de la zona minera de Moa (Cuba): influencia del comportamiento hidromecánico. Tesis doctoral. Universidad Politécnica de Cataluña (UPC), Barcelona, España.

UNI (Unión de Empresas del Níquel) (1994). Informe acerca de los principales focos de contaminación del medio ambiente como resultado del impacto de la industria del níquel. Política aplicada en la solución de estos problemas. Documento interno. 8 p.

Recibido: julio 2020

Revisado: diciembre 2020

Aceptado: enero 2021

Publicado: diciembre 2021 